\title{
REMARKS ON SYZYGIES OF $d$-GONAL CURVES
}

\author{
MARIAN APRODU
}

\begin{abstract}
We apply a degenerate version of a result due to Hirschowitz, Ramanan and Voisin to verify Green and Green-Lazarsfeld conjectures over explicit open sets inside each $d$-gonal stratum of curves $X$ with $d<\left[g_{X} / 2\right]+2$. By the same method, we verify the Green-Lazarsfeld conjecture for any curve of odd genus and maximal gonality. The proof invokes Voisin's solution to the generic Green conjecture as a key argument.
\end{abstract}

\section{Introduction}

A result due to Green and Lazarsfeld, cf. [G, Appendix], shows that the existence of special linear systems on a complex projective variety $X$ reflects into non-vanishing of certain Koszul cohomology groups $K_{p, 1}$. Recall that for two integers $p$ and $q$ and a line bundle $L$ on $X$, the Koszul cohomology group $K_{p, q}(X, L)$ was defined in $[\mathrm{G}]$ as the cohomology of the complex:

$$
\wedge^{p+1} H^{0}(L) \otimes H^{0}\left(L^{q-1}\right) \rightarrow \wedge^{p} H^{0}(L) \otimes H^{0}\left(L^{q}\right) \rightarrow \wedge^{p-1} H^{0}(L) \otimes H^{0}\left(L^{q+1}\right) .
$$

On a smooth, connected curve $X$, one expects that the Green-Lazarsfeld nonvanishing result be revertible, that is non-trivial $K_{p, 1}$ groups with values in line bundles chosen in convenient ways would give rise to special linear systems on the curves. This amounts to proving that particular Koszul cohomology groups vanish if some linear series loci are empty.

For the canonical bundle, Green conjectured that $K_{g-c-1,1}\left(X, K_{X}\right)=0$ for any curve $X$ of genus $g$ and Clifford index $c$, see [G]. Similarly, if $X$ is of gonality $d$, Green and Lazarsfeld predicted $K_{h^{0}(L)-d, 1}(X, L)=0$ for any line bundle $L$ of sufficiently large degree, see [GL1]. Put in other words, the two basic invariants of a curve, Clifford index and gonality, would be read off suitable Koszul cohomology groups. It is important to mention that they are related by the inequalities $d-3 \leq c \leq d-2 \leq\left[\left(g_{X}-1\right) / 2\right]$, cf. [ACGH] and [CM].

Both Green, and Green-Lazarsfeld conjectures have been verified for general curves of any genus (for Green's conjecture see [V2] and [V3], and for the GreenLazarsfeld conjecture we refer to $[\mathrm{AV}]$ and $[\mathrm{A} 2])$ with the notable difference that

Received December 7, 2004.

2000 Mathematical Subject Classification. 14H51, 14C20, 13D02.

Key words and phrases: syzygies, curve, gonality, Clifford index, stable curve.

This work was supported by a Humboldt Research Fellowship. 
in the odd-genus case, the Green conjecture is known to hold for any curve of maximal gonality, as shown by Hirschowitz, Ramanan and Voisin, $c f$. [HR] and [V3].

Theorem 1 (Hirschowitz-Ramanan-Voisin). Any smooth curve $X$ of genus $g=$ $2 k+1 \geq 5$ with $K_{k, 1}\left(X, \omega_{X}\right) \neq 0$ carries a pencil of degree $k+1$.

The proof of this remarkable result relies on a comparison of two divisors in the moduli space $\mathscr{M}_{2 k+1}$, the one of curves which carry a $g_{k+1}^{1}$ and, respectively, the locus of canonical curves with extra-syzygies. Already showing that the latter one is a genuine divisor, and does not cover the whole moduli space is hard work, and was concluded only recently in Voisin's tour de force [V2], [V3], after having developed a completely new approach to the problem in [V2].

The Green conjecture is known to hold also for curves of non-maximal gonality which are generic in their gonality strata, and this happens for all possible gonalities $c f$. [V2, Corollary 1] and [T, Theorem 0.4], see also [S1, Theorem]. It is well-known that by fixing the gonality $d$ we obtain a stratification of the moduli space of curves with irreducible gonality strata, the maximal possible value for $d$ corresponding to the open stratum. Therefore, it makes perfect sense to speak about general points in a given gonality stratum.

Starting from the generic Green conjecture, Voisin had the idea to degenerate smooth curves on a $K 3$ surface to irreducible nodal curves, in the same linear system, in order to make the Koszul cohomology of the latter ones vanish, and so she verified the Green conjecture for the normalizations of these curves, $c f$. [V2]. This fact resulted into a very short and elegant solution for the Green conjecture for generic curves $X$ of non-maximal gonality larger than $g_{X} / 3$. Then she observed furthermore that exactly the same method yields, in completion to [A1, Theorem 1], to a solution for the Green-Lazarsfeld conjecture for generic curves of fixed gonality in the same order range, $c f$. [AV, Theorem 1.3] and [AV, Theorem 1.4]. This strategy did much better than the partial [A1, Theorem 3], see $[\mathrm{AV}$, Remark 2] for some related comments.

At the other end of the spectrum, normalizations of irreducible nodal curves on $\mathbb{P}^{1} \times \mathbb{P}^{1}$ have been used to verify the two conjectures for generic curves $X$ in any stratum of gonality bounded in the order $\sqrt{g_{X}}, c f$. [S1, Theorem], and [A1, Theorem 4], the vanishing of the Koszul cohomology having been proved, in contrast to Voisin, directly on the normalizations. Further connections between Green and Green-Lazarsfeld conjectures are emphasized in [A1, Appendix and II].

Having had these indications pleading for unity, it seems natural to try to find a common space of curves with fixed gonality on which the two conjectures be treated in a unitary way - this is the goal of the present work. We use degenerations, taking the path shown by Voisin, which is to compute the Koszul cohomology directly on nodal curves, instead of normalizing them first. The proof of our first result will show that this is a very natural thing to do. 
Theorem 2. Let $d \geq 3$ be an integer, and $X$ be a smooth $d$-gonal curve with $d<$ $\left[g_{X} / 2\right]+2$, and such that $\operatorname{dim}\left(W_{d+n}^{1}(X)\right) \leq n$ for all $0 \leq n \leq g_{X}-2 d+2$. Then $\operatorname{Cliff}(X)=d-2$, and $X$ verifies both Green, and Green-Lazarsfeld conjectures.

In the statement, $W_{d+n}^{1}(X)$ denotes the subvariety of $\operatorname{Pic}_{d+n}(X)$ of bundles with two or more independent sections. We mention that, since $X$ is nonhyperelliptic, we always have the bound $\operatorname{dim}\left(W_{d+n}^{1}(X)\right) \leq d+n-3$ for all $0 \leq$ $n \leq g_{X}-2 d+2$, see [HMa]. Beside, according to a problem raised by G. Martens, $c f$. [GMa, Statement (T), p. 280], it is very likely that the conditions appearing in the statement of Theorem 2 be reduced to the single condition $\operatorname{dim}\left(W_{d}^{1}(X)\right)=$ 0 . The locus of curves satisfying the hypotheses of the theorem is anyway nonempty and open in the $d$-gonal stratum, and all but one possible gonality strata are concerned. In particular, Theorem 2 gives an alternate proof to [T, Theorem 0.4], [S1, Theorem], [A1, Theorem 4], and, for the Green-Lazarsfeld conjecture, it fills in the existing gap for generic $d$-gonal curves with $d$ in order between $\sqrt{g_{X}}$ and $g_{X} / 3$.

For small $d$ one can specify the curves which do not satisfy the hypothesis, $c f$. $[\mathrm{ACGH}],[\mathrm{HMa}],[\mathrm{Mu}],[\mathrm{Ke}]$. Among the new effective results that follow directly from Theorem 2 we count then the validity of the Green-Lazarsfeld conjecture for non-bielliptic tetragonal curves, and of the two conjectures for pentagonal and for hexagonal curves which are neither trielliptic nor double coverings of curves of genus three, see Corollary 10 in Section 3.

The extremal case $g_{X}-2 d+2=0$ indicates that both Green, and GreenLazarsfeld conjectures are verified for any curve $X$ of even genus and maximal gonality which carries finitely many minimal pencils. From the Green conjecture point of view, this can be seen as a first step in finding a correspondent of the Hirschowitz-Ramanan-Voisin Theorem in the even-genus case. Other consequence of Theorem 2 regards curves of even genus and maximal Clifford index on $K 3$ surfaces, $c f$. Corollary 11.

The case not covered by Theorem 2 is, as expected, the case of curves of odd genus and maximal gonality. The second result, which refines [A2, Theorem 1.1], is concerned with the Green-Lazarsfeld conjecture for these curves, for which the Green conjecture has already been settled.

Theorem 3. The Green-Lazarsfeld conjecture is valid for any smooth curve $X$ of genus $g_{X}=2 k-1$ and gonality $k+1$, with $k \geq 3$.

We obtain directly from Theorem 3 a version of the Hirschowitz-RamananVoisin Theorem for syzygies of pluricanonical curves.

Corollary 4. If $X$ is a smooth curve of genus $2 k-1$, where $k \geq 3$, with

$$
K_{2(2 p-1)(k-1)-(k+1), 1}\left(X, \omega_{X}^{\otimes p}\right) \neq 0
$$

for some $p \geq 2$, then $X$ carries a $g_{k}^{1}$.

Roughly speaking, the proof strategy of the two theorems above is the same, namely to construct, starting from $X$, some suitable singular stable curves of 
genus $g=2 k+1$ and analyze their syzygies. A degenerate version of the Hirschowitz-Ramanan-Voisin Theorem, proved in Section 2, tells us that if these stable curves have extra-syzygies, then they are limits of smooth curves with extra-syzygies, and this fact translates into the existence of special torsion-free sheaves of rank one, which will trace pencils on the original curve $X$. For Theorem 2, the singular curves which we construct are irreducible, and this choice was inspired directly by the ideas of Voisin applied in [V2] and [AV], and which we have briefly explained above. This result is then a natural continuation of [V2, Corollary 1] and [AV, Theorems 1.3 and 1.4]. The difference is that we work now with abstract families of curves degenerating to a stable curve, whereas in [V2] and $[\mathrm{AV}]$, these degenerations were always made on $K 3$ surfaces. The freedom we gain also reflects into the possibility of working with curves with arbitrarily many nodes, getting rid of the main obstruction to improving the statements quoted above. As for Theorem 3, the singular curves which we construct are curves with one smooth rational component, similarly to [A2, Theorem 1.1]. These curves are themselves limits of irreducible curves with two nodes, indicating this case as being "more degenerate" than the others, and giving a hint of why should it be treated separately. The origin of this difference is that this is the only case of a gonality stratum whose general members carry infinitely many minimal pencils.

\section{Syzygies of singular stable curves with very ample canonical bundle}

Throughout this section the arithmetic genus will remain fixed to $g=2 k+1$, where $k \geq 2$. We study the Koszul cohomology of a stable curve $Y$ of genus $g$ in relation with its geometry. Recall that stable curves are reduced connected curves with finite group of automorphisms, and with only simple double points (nodes) as possible singularities. They have been introduced by Deligne and Mumford with the aim of compactifying the moduli space $\mathscr{M}_{g}$ of smooth curves of genus $g$. Singular stable curves of arithmetic genus $g$ lie on a normal-crossing divisor $\Delta_{0} \cup \cdots \cup \Delta_{[g / 2]}$ in $\overline{\mathscr{M}}_{g}$, on the boundary of $\mathscr{M}_{g}$, and the general element in $\Delta_{0}$ is irreducible, whereas a general element in $\Delta_{i}$ is the union of two curves of genus $i$ and $g-i$ respectively, meeting in one point.

From now on, we shall work exclusively with stable curves with very ample canonical bundle, see $[\mathrm{C}$, Theorem F] and [CFHR, Theorem 3.6] for precise criteria.

Notation. The open subspace in $\overline{\mathscr{M}}_{g}$ of points corresponding to stable curves of genus $g$ with very ample canonical bundle, which can be easily shown to be contained in $\mathscr{M}_{g} \cup \Delta_{0}$, will be denoted by $\mathscr{M}_{g}^{\mathrm{va}}$.

Let $[Y] \in \mathscr{M}_{g}^{\text {va }}$, and denote, for simplicity $\mathbb{P}:=\mathbb{P} H^{0}\left(Y, \omega_{Y}\right)^{*}$, which contains the image of $Y$, set $Q=T_{\mathbb{P}}(-1)$ the universal quotient bundle, and $Q_{Y}$ the restriction of $Q$ to $Y$. The Koszul cohomology of $Y$ with values in $\omega_{Y}$ has then the following description, [HR, Proposition 2.1]. 
Proposition 5 (Hirschowitz-Ramanan). With the notation above, for any $n \geq$ 1 , there exists an isomorphism

$$
K_{n, 1}\left(Y, \omega_{Y}\right) \cong \operatorname{ker}\left(H^{0}\left(\mathbb{P}, \wedge^{2 k-n+1} Q(1)\right) \rightarrow H^{0}\left(Y, \wedge^{2 k-n+1} Q_{Y} \otimes \omega_{Y}\right)\right) .
$$

Remark 6. For the choice $n=k$ and for a smooth curve $Y$, Hirschowitz and Ramanan remarked that the two spaces appearing in the description above, namely $H^{0}\left(\mathbb{P}, \wedge^{k+1} Q(1)\right)$ and $H^{0}\left(Y, \wedge^{k+1} Q_{Y} \otimes \omega_{Y}\right)$, have the same dimension, cf. [HR, Proof of Proposition 3.1]. This observation was essential in the proof of their main result. Applying the Riemann-Roch Theorem, and the Serre duality we observe that the two spaces in question are still of the same dimension even if $Y$ was a singular stable curve with very ample canonical bundle, since $H^{0}\left(Y, \wedge^{k+1} Q_{Y}^{*}\right)=0$. The vanishing of $H^{0}\left(Y, \wedge^{m+1} Q_{Y}^{*}\right)$ for any $m \geq 0$ is a standard fact, and is implied by the following classical remarks. Firstly, we know that there are natural exact sequences, for any $p$ and $q$,

$0 \rightarrow H^{0}\left(Y, \wedge^{p+1} Q_{Y}^{*} \otimes \omega_{Y}^{q-1}\right) \rightarrow \wedge^{p+1} H^{0}\left(Y, \omega_{Y}\right) \otimes H^{0}\left(Y, \omega_{Y}^{q-1}\right) \rightarrow H^{0}\left(Y, \wedge^{p} Q_{Y}^{*} \otimes \omega_{Y}^{q}\right)$.

Secondly, the Koszul differential $\wedge^{m+1} H^{0}\left(\omega_{Y}\right) \rightarrow \wedge^{m} H^{0}\left(\omega_{Y}\right) \otimes H^{0}\left(\omega_{Y}\right)$ is injective, and it factors through the inclusion of $H^{0}\left(\wedge^{m} Q_{Y}^{*} \otimes \omega_{Y}\right)$ into $\wedge^{m} H^{0}\left(Y, \omega_{Y}\right) \otimes$ $H^{0}\left(Y, \omega_{Y}\right)$.

Convention. Hirschowitz and Ramanan used the term with extra-syzygies to designate a smooth curve $Y$ of genus $2 k+1$ with $K_{k, 1}\left(Y, \omega_{Y}\right) \neq 0$. We adopt this terminology and extend it to singular curves with the same non-vanishing property.

From the syzygy point of view, singular stable curves with very ample canonical bundle behave similarly to smooth curves. For instance, those which have extra-syzygies, are degenerations of smooth curves with extra-syzygies.

Proposition 7. Let $Y$ be a singular stable curve of genus $g=2 k+1$ with very ample canonical bundle. Then $Y$ has extra-syzygies if and only if $[Y]$ belongs to the closure of the locus of $(k+1)$-gonal smooth curves.

Proof. We set some notation first. Following [HM], let $D_{k+1}$ be the divisor on $\mathscr{M}_{g}$ of curves with a pencil of degree $k+1$, let $\bar{D}_{k+1}$ be its closure in $\overline{\mathscr{M}}_{g}$, $D_{k+1}^{\mathrm{va}}=\bar{D}_{k+1} \cap \mathscr{M}_{g}^{\mathrm{va}}$, and $\Delta_{0}^{\mathrm{va}}=\Delta_{0} \cap \mathscr{M}_{g}^{\mathrm{va}}$.

By semi-continuity, the locus of curves in $\mathscr{M}_{g}^{\mathrm{va}}$ with extra-syzygies is closed. Similarly to $[\mathrm{HR}]$, we show that it is actually a divisor. It amounts to proving that its inverse image on a covering $S^{\mathrm{va}} \rightarrow \mathscr{M}_{g}^{\mathrm{va}}$ is a divisor. We choose a smooth $S^{\mathrm{va}}$ on which an universal curve exists. From [HR, Proof of Proposition 3.1] and from Remark 6 it follows that the locus of points on $S^{\mathrm{va}}$ corresponding to curves with extra-syzygies is the degeneracy locus of a morphism of vector bundles of the same rank. Then this locus is a divisor, since we know it is not the whole space, $c f$. [V3, Theorem 4].

Back to $\mathscr{M}_{g}^{\mathrm{va}}$, the computation of [HR] shows that the divisor of curves with extra-syzygies is equal to a multiple of $D_{k+1}^{\mathrm{va}}$ plus, eventually, a multiple of $\Delta_{0}^{\mathrm{va}}$ 
(recall that $\Delta_{0}$ is irreducible, and so is $\Delta_{0}^{\mathrm{va}}$ ). The possibility that the whole $\Delta_{0}^{\mathrm{va}}$ be contained in the locus of curves with extra-syzygies is ruled out by the same result of [V3]. The example of a curve in $\Delta_{0}^{\mathrm{va}}$ with no extra-syzygies given by Voisin, and used in [AV, Proof of Theorem 1.4], is an irreducible curve with one node lying on a $K 3$ surface, compare to the proof of Theorem 2 in the next section. In particular, it follows that a curve in $\Delta_{0}^{\mathrm{va}}$ has extra syzygies if and only if it belongs to $D_{k+1}^{\mathrm{va}}$, which we wanted to prove.

We analyze next further consequences of having extra-syzygies, and show that this condition yields to the existence of certain suitable sheaves. By means of Proposition 7 , a singular stable curve $Y$ with $\omega_{Y}$ very ample and with extrasyzygies lies in an one-dimensional flat family $\mathscr{C} \rightarrow T$ of curves such that $\mathscr{C}_{t_{0}} \cong$ $Y$, and $\mathscr{C}_{t}$ are smooth, and belong to $D_{k+1}$ for $t \neq t_{0}$. For safety, let us make the further assumption that $Y$ has trivial automorphism group, although this extra-condition might be unnecessary. By shrinking $T$ if needed, we can make the same hypothesis for the curves $\mathscr{C}_{t}$. By the compactification theory of the generalized relative Jacobian, see $[\mathrm{Ca}]$ and $[\mathrm{P}]$, there exists a family $\mathscr{J}_{1-k}(\mathscr{C} / T)$, flat and proper over $T$, whose fiber over $t \neq t_{0}$ is just the Jacobian variety of line bundles of degree $k+1$, whereas the fiber over $t_{0}$ parametrizes $g r$-equivalence

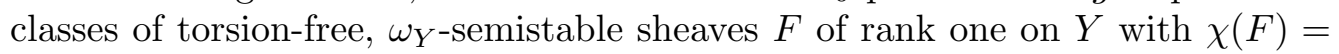
$1-k$; by definition, a coherent sheaf on $Y$ is torsion-free if it has no non-zero subsheaf with zero-dimensional support. It follows that the subspace of pairs $\left\{\left(\mathscr{F}_{t}, \mathscr{C}_{t}\right) \in \mathscr{J}_{1-k}(\mathscr{C} / T) \times_{T} \mathscr{C}, h^{0}\left(\mathscr{C}_{t}, \mathscr{F}_{t}\right) \geq 2\right\}$ is closed in the fibered product, and, since $\left[\mathscr{C}_{t}\right] \in D_{k+1}$ for all $t \neq t_{0}$, we are lead to the following degenerate version of the Hirschowitz-Ramanan-Voisin Theorem.

Proposition 8. Let $Y$ be a singular stable curve of genus $g=2 k+1$ with very ample canonical bundle and trivial automorphism group. If $Y$ has extra-syzygies, then there exists a torsion-free, $\omega_{Y}$-semistable sheaf $F$ of rank one on $Y$ with $\chi(Y, F)=1-k$ and $h^{0}(Y, F) \geq 2$.

In several particular cases, this sheaf can be analyzed even further to deduce the existence of other suitable linear systems on the normalizations of components of $Y$, see the proofs of Theorems 2 and 3, and compare to [HM, Corollary 1, p. 68].

\section{Proof of Theorem 2 and consequences}

We recall the following result from [A1], which will be used for the GreenLazarsfeld conjecture, see also [AV, Theorem 2.1]:

Theorem 9. If $L$ is a nonspecial line bundle on a smooth curve $X$, which satisfies $K_{n, 1}(X, L)=0$, for an integer $n \geq 1$, then, for any effective divisor $E$ of degree $e \geq 1$, one has $K_{n+e, 1}(X, L+E)=0$.

Proof of Theorem 2. We set some notation. Define $k=g_{X}-d+1 \geq 1$ and $\nu=g_{X}-2 d+2 \geq 0$, so that $d=k-\nu+1, g_{X}=2 k-\nu$, and $0 \leq n \leq \nu$. 
One should ensure firstly that curves satisfying the hypothesis of the theorem exist. In the case $\nu=0$, this is automatic, but in the other cases it is less clear. We assume therefore that $\nu \geq 1$, condition which is equivalent to $d<g_{X} / 2+1$, and adopt the notation of $[\mathrm{AC}]$. From $[\mathrm{AC}$, Theorem (2.6)], we know that a general $d$-gonal curve $X$ has precisely one minimal pencil, and moreover, any other base-point-free $g_{h}^{1}$ on $X$ with $h<g_{X} / 2+1$ is composed with the given $g_{d}^{1}$. We count now the possible dimensions of the irreducible components of the varieties $G_{d+n}^{1}(X)$ parametrizing the $g_{d+n}^{1}$ 's on a generic $d$-gonal curve $X$, and prove that they cannot exceed $n$. Since $G_{d+n}^{1}(X)$ is the canonical blowup of the $W_{d+n}^{1}(X)$ endowed with the determinantal structure, [ACGH, Chapter IV.3], it would prove our existence claim.

Let $S$ be a smooth variety covering $\mathscr{M}_{2 k-\nu}$ on which there exists an universal curve $\mathscr{C} \rightarrow S$, let $\mathscr{G}_{d}^{1}$, and $\mathscr{G}_{d+n}^{1}$ the varieties over $S$ parameterizing pairs of smooth curves of genus $2 k-\nu$, and pencils of degree $d$, and respectively $d+n$, and let $\mathscr{X}$ be an irreducible component of $\mathscr{G}_{d}^{1} \times_{S} \mathscr{G}_{d+n}^{1}$ which dominates $\mathscr{G}_{d}^{1}$. Since $\mathscr{G}_{d}^{1}$ is birational to the locus of $d$-gonal curves, $c f$. [AC, Theorem (2.6)] its dimension equals $2(2 k-\nu)+2 d-5$. Therefore, we must prove that $\operatorname{dim}(\mathscr{X}) \leq$ $2(2 k-\nu)+2 d+n-5$, which would ensure that a general fibre $\mathscr{X}_{\xi}$ of the projection $\mathscr{X} \rightarrow \mathscr{G}_{d}^{1}$ is of dimension at most $n$.

Let us suppose first that the general element of $\mathscr{X}$ corresponds to a smooth $d$ gonal curve $X$ with a base-point-free $g_{d+n}^{1}$ which is not composed with a rational involution. Since $\mathscr{X}$ dominates $\mathscr{G}_{d}^{1}$, and minimal pencils on general $d$-gonal curves are simple, the hypotheses of [AC, Proposition (2.4)] are fulfilled. Coupled with [AC, Theorem (2.6)], it implies that either $n=0$, and the two $g_{d}^{1}$ 's for a generic point in $\mathscr{X}$ coincide, in which case the projection from $\mathscr{X}$ to $\mathscr{G}_{d}^{1}$ is birational, or $d+n \geq(2 k-\nu) / 2+1$ and then $\operatorname{dim}(\mathscr{X})=(2 k-\nu)+2 d+2(d+$ $n)-7 \leq 2(2 k-\nu)+2 d+n-5$.

The other components have the following description. Suppose that there is a non-empty open subspace $\mathscr{U}$ of $\mathscr{X}$ whose points correspond to smooth $d$-gonal curves together with a $g_{d+n}^{1}$ having precisely $\mu$ base-points, where $0 \leq \mu \leq n$. Then there is a natural map $\mathscr{U} \rightarrow \mathscr{G}_{d}^{1} \times{ }_{s} \mathscr{G}_{d+n-\mu}^{1}$, obtained by erasing the base points of the corresponding $g_{d+n}^{1}$ 's. Since $\mathscr{U}$ is irreducible, its image is contained in an irreducible component $\mathscr{Y}$ of $\mathscr{G}_{d}^{1} \times_{S} \mathscr{G}_{d+n-\mu}^{1}$, whose generic point corresponds to a $d$-gonal curve together with a base-point-free $g_{d+n-\mu}^{1}$ (recall that base-point-freeness is an open condition). The projections on $\mathscr{G}_{d}^{1}$ commute with the corestriction $\mathscr{U} \rightarrow \mathscr{Y}$, so that $\mathscr{Y}$ dominates $\mathscr{G}_{d}^{1}$, too. If one denotes $\mathscr{C}^{(\mu)}=\left(\mathscr{C} \times_{S} \cdots \times_{S} \mathscr{C}\right) / \mathfrak{S}_{\mu} \rightarrow S$, where the product was taken $\mu$ times, the relative symmetric product, then the induced map $\mathscr{U} \rightarrow \mathscr{Y} \times{ }_{S} \mathscr{C}^{(\mu)}$ defined by splitting the corresponding $g_{d+n}^{1}$ 's into their free and fixed parts respectively, is injective. In particular, $\operatorname{dim}(\mathscr{X}) \leq \operatorname{dim}(\mathscr{Y})+\mu$. We prove next that $\operatorname{dim}(\mathscr{Y}) \leq$ $2(2 k-\nu)+2 d+n-\mu-5$. If the general element of $\mathscr{Y}$ corresponds to a smooth $d$-gonal curve $X$ with a base-point-free $g_{d+n-\mu}^{1}$ which is not composed with a rational involution, this inequality follows directly from what we have said above. 
Let us suppose then that a general point of $\mathscr{Y}$ corresponds to a smooth $d$-gonal curve together with base-point-free $g_{d+n-\mu}^{1}$ 's which is composed with a rational involution of given degree $\gamma \geq 2$. Then there exists $0 \leq m<n$ such that $d+n-\mu=\gamma(d+m)$, and there exists also a dominant map $\mathscr{Z} \times G_{\gamma}^{1}\left(\mathbb{P}^{1}\right) \rightarrow \mathscr{Y}$ where $\mathscr{Z}$ is an irreducible component of $\mathscr{G}_{d}^{1} \times{ }_{S} \mathscr{G}_{d+m}^{1}$ whose general member is a $d$-gonal curve endowed with a base-point-free $g_{d+m}^{1}$ which is not composed with a rational involution. This morphism maps a 4-tuple $\left(X, f, f^{\prime}, \lambda\right)$ with $f$ a $g_{d}^{1}$ on $X, f^{\prime}$ a $g_{d+m}^{1}$ on $X$, and $\lambda$ a covering $\mathbb{P}^{1} \rightarrow \mathbb{P}^{1}$ of degree $\gamma$, to $\left(X, f, \lambda \circ f^{\prime}\right)$. Again, $\mathscr{Z}$ dominates $\mathscr{G}_{d}^{1}$, which implies $\operatorname{dim}(\mathscr{Z}) \leq 2(2 k-\nu)+2 d+m-5$, and furthermore $\operatorname{dim}(\mathscr{Y}) \leq 2(2 k-\nu)+2 d+m-5+2 \gamma-2 \leq 2(2 k-\nu)+2 d+n-\mu-5$.

We consider next $(\nu+1)$ pairs of distinct points $\left(x_{i}, y_{i}\right)$, with $0 \leq i \leq \nu$ such that for any choice of $(n+1)$ pairs among them, $\left(x_{i_{j}}, y_{i_{j}}\right)$, with $0 \leq j \leq n$ and $0 \leq n \leq \nu$, there exists no $L_{n} \in W_{d+n}^{1}(X)$ such that $h^{0}\left(X, L_{n}\left(-x_{i_{j}}-y_{i_{j}}\right)\right) \geq 1$ for all $0 \leq j \leq n$. The $(\nu+1)$-tuple of cycles $\left(x_{0}+y_{0}, \ldots, x_{\nu}+y_{\nu}\right)$ can be chosen to be generic in the space $X^{(2)} \times \cdots \times X^{(2)}$. This is allowed by the easy observation that, for any $n$, the incidence variety

$$
\left\{\left(x_{0}+y_{0}, \ldots, x_{n}+y_{n}, L_{n}\right), h^{0}\left(L_{n}\left(-x_{i}-y_{i}\right)\right) \geq 1 \text { for all } i\right\}
$$

is at most $(2 n+1)$-dimensional, whereas $\operatorname{dim}\left(X^{(2)} \times \cdots \times X^{(2)}\right)=2 n+2$. Indeed, the incidence variety in question is covered by the similar incidence variety $\Xi$ inside $X^{2 n+2} \times W_{d+n}^{1}(X)$, and the fibers of the projection map from $\Xi$ to $X^{n+1}$ obtained by erasing the bundle $L_{n}$ and the $y$ 's are finite covers of $W_{d+n}^{1}(X)$ via the restriction of the canonical projection.

Then we construct an irreducible curve $Y$ obtained by identifying $x_{i}$ to $y_{i}$ for all $i$, and denote by $p_{i}$ the corresponding node of $Y$, and by $f: X \rightarrow Y$ the normalization morphism. From the genericity of the cycles $x_{i}+y_{i}$, the curve $Y$ can be considered to be free from non-trivial automorphisms and with very ample canonical bundle, apply [C, Theorem F], [CFHR, Theorem 3.6].

We prove first that $K_{k, 1}\left(Y, \omega_{Y}\right)=0$. Suppose that $K_{k, 1}\left(Y, \omega_{Y}\right) \neq 0$. From Proposition 8, we obtain a torsion-free sheaf $F$ of rank one on $Y$ with $\chi(F)=$ $1-k$, and $h^{0}(F) \geq 2$. The sheaf $F$ is either a line bundle, or the direct image of a line bundle on a partial normalization of $Y$. Observe that this partial normalization cannot be $X$ itself. Indeed, if $F=f_{*} L$ with $L$ a line bundle on $X$, then $\chi(L)=\chi(F)=1-k$, and $h^{0}(L)=h^{0}(F) \geq 2$, which means that $L$ is a $g_{d-1}^{1}$ on $X$, contradicting the hypothesis. Let us consider then $\varphi: Z \rightarrow Y$ the normalization of the $(\nu-n)$ points $p_{n+1}, \ldots, p_{\nu}$, for some $0 \leq n \leq \nu$. Let furthermore $\psi: X \rightarrow Z$ be the normalization of the remaining $(n+1)$ points $p_{0}, \ldots, p_{n}$, and suppose $F=\varphi_{*} L$, for a line bundle $L$ on $Z$. Under these assumptions, we obtain $\chi(L)=\chi(F)=1-k$, and so $\chi\left(\psi^{*} L\right)=2-k+n$, which implies that $\operatorname{deg}\left(\psi^{*} L\right)=d+n$. Beside, $\psi^{*} L$ has at least two independent sections. Since for any node $p_{i}$ with $0 \leq i \leq n$ there is a non-zero section of $F$ vanishing at $p_{i}$, it follows that $h^{0}\left(X,\left(\psi^{*} L\right)\left(-x_{i}-y_{i}\right)\right) \geq 1$ for all $0 \leq i \leq n$, which contradicts the choice we made. 
We proved $K_{k, 1}\left(Y, \omega_{Y}\right)=0$. To conclude, we apply [AV, Lemma 2.3] and Voisin's Remark [V2, p. 367], and have $K_{k, 1}\left(X, K_{X}\right) \subset K_{k, 1}\left(X, K_{X}+x_{i}+y_{i}\right) \subset$ $K_{k, 1}\left(Y, \omega_{Y}\right)$, for all $i$. We obtain thence the vanishing of $K_{k, 1}\left(X, K_{X}\right)$ and of $K_{k, 1}\left(X, K_{X}+x_{i}+y_{i}\right)$, for all $i$. The vanishing $K_{k, 1}\left(X, K_{X}\right)=0$ is the statement of the Green conjecture for $X$, the fact that $\operatorname{Cliff}(X)$ equals $d-2$ being implied by the Green-Lazarsfeld non-vanishing theorem $c f$. [G, Appendix]. The vanishing $K_{k, 1}\left(X, K_{X}+x_{i}+y_{i}\right)=0$ is precisely the one predicted by the Green-Lazarsfeld conjecture for the bundle $K_{X}+x_{i}+y_{i}$. Then apply Theorem 9 to conclude that the Green-Lazarsfeld conjecture is verified for any line bundle of degree at least $3 g_{X}$ on $X$, compare to [AV, Remark 1].

For small $d$, one can employ classical results due H. Martens, Mumford and Keem on the dimensions of the Brill-Noether loci, cf. [ACGH], [HMa], [Mu], $[\mathrm{Ke}]$ to obtain the following.

Corollary 10. Let $X$ be a non-hyperelliptic smooth curve of gonality $d \leq 6$, with $d<\left[g_{X} / 2\right]+2$, and suppose that $X$ is not one of the following: plane curve, bielliptic, triple cover of an elliptic curve, double cover of a curve of genus three, hexagonal curve of genus 10 or 11 . Then $\operatorname{Cliff}(X)=d-2$ and $X$ verifies both Green, and Green-Lazarsfeld conjectures.

Proof. For a trigonal curve $X$, one has to prove that $\operatorname{dim}\left(W_{n+3}^{1}(X)\right) \leq n$ for all $0 \leq n \leq g_{X}-4$. This follows from [HMa, Theorem 1], as we know that $\operatorname{dim}\left(W_{n+3}^{1}(X)\right) \leq n+1$ and the equality is never achieved, since $X$ is nonhyperelliptic. If $d=4$, one has to prove $\operatorname{dim}\left(W_{n+4}^{1}(X)\right) \leq n$ for all $0 \leq n \leq$ $g_{X}-6$. In this case, we apply Mumford's refinement to the Theorem of $\mathrm{H}$. Martens, cf. $[\mathrm{Mu}]$, which shows that $\operatorname{dim}\left(W_{n+4}^{1}(X)\right) \leq n+1$, and equality could eventually hold only for trigonal (which we excluded), bielliptic curves or smooth plane quintics. The other cases $d=5$ and $d=6$ are similar, and follow from [Ke, Theorem 2.1], and [Ke, Theorem 3.1], respectively.

Some cases in the Corollary 10 were known before, others are new. For trigonal curves, Green's conjecture was known to hold from Enriques and Petri, and the Green-Lazarsfeld conjecture was verified by Ehbauer [Eh]. For tetragonal curves, we knew that the Green conjecture was valid, cf. [S2], and [V1]. All the other cases seem to be new. Plane curves, which were excepted from our statement, also verify the two conjectures, cf. [Lo], and [A1]. Note that in a number of other cases for which our result does not apply, Green's conjecture is nonetheless satisfied, for instance, for hexagonal curves of genus 10 and Clifford index 3 , complete intersections of two cubics in $\mathbb{P}^{3}$, see [Lo].

For large $d$ we cannot give similar precise results, but we still obtain a number of examples for which Theorem 2 can be applied. For instance, curves of even genus which are Brill-Noether-Petri generic satisfy the hypothesis of Theorem 2, so they verify the two syzygy conjectures. Other cases are obtained by looking at curves on some surfaces, when the special geometry of the pair (curve, surface) is used, as in the following. 
Corollary 11. Let $X$ be a smooth curve of genus $2 k$ and maximal Clifford index $k-1$, with $k \geq 2$ abstractly embedded in a $K 3$ surface. Then $X$ verifies the Green conjecture.

Proof. Since the Clifford index of $X$ is maximal, and Clifford index is constant in the linear system of $X, c f$. [GL2], the gonality is also maximal, and thus constant for smooth curves in $|X|$. Then the hypotheses of [CP, Lemma 3.2 (b)] are verified, which implies that a general smooth curve in the linear system $|X|$ has only finitely many pencils of degree $k+1$. From Theorem 2 it follows that the Green conjecture is verified for a general smooth curve $C \in|X|$, that is $K_{k, 1}\left(C, K_{C}\right)=0$. By applying Green's hyperplane section theorem [G, Theorem (3.b.7)] twice, we obtain $K_{k, 1}\left(X, K_{X}\right)=0$, which means that $X$ satisfies Green's conjecture, too.

Note that Corollary 11 does not apply to the particular curves considered by Voisin in [V2], [V3], as they are implicitly used in the proof.

In view of Theorem 2 and [HMa, Statement (T)], it seems that understanding the geometry of curves which carry infinitely many minimal pencils plays a crucial role in the quest for a complete solution to the two conjectures. The problem of studying these curves has already been raised by Eisenbud, Lange, G. Martens and Schreyer, [ELMS, Remark 3.8]. For the beginning, it would be interesting to know whether the locus of smooth curves of even genus $2 k$ with maximal Clifford index and infinitely many $g_{k+1}^{1}$ 's is non-empty.

\section{Proof of Theorem 3}

Proof of Theorem 3. We start by noting that $\operatorname{dim}\left(W_{k+1}^{1}(X)\right)=1$, see [FHL], [ACGH, Lemma IV.(3.3) p. 181 and Ex. VII.C-2, p. 329]. Then one can find three distinct points $x, y$ and $z$ of $X$ which do not belong at the same time to a pencil of degree $k+1$. As in the previous proof, the cycle $x+y+z$ can be generically chosen in $X^{(3)}$, since in our case the incidence variety $\{(x+y+z, L) \in$ $\left.X^{(3)} \times W_{k+1}^{1}(X), h^{0}(L(-x-y-z)) \geq 1\right\}$ is two-dimensional, so that the image of its projection to $X^{(3)}$ is a surface.

For these three points, we shall prove that $K_{k, 1}\left(X, K_{X}+x+y+z\right)=0$, which will imply, by means of Theorem 9, that the Green-Lazarsfeld conjecture is verified for any line bundle of degree at least $3 g_{X}+1$ on $X$, compare to [A2, Remark 2.6].

We suppose to the contrary that $K_{k, 1}\left(X, K_{X}+x+y+z\right) \neq 0$, and shall reach a contradiction. For this aim, we adapt the arguments already used in the proof of Theorem 2 to this new situation. We introduce a curve $Y$ with two irreducible components: the first one is $X$, and the second one is a smooth rational curve $E$ which passes through the points $x, y$ and $z$. Then $Y$ is a stable curve of arithmetic genus $g=2 k+1$, and $K_{k, 1}\left(Y, \omega_{Y}\right) \cong K_{k, 1}\left(X, K_{X}+x+y+z\right)$, compare to [A2, Proof of Lemma 2.4]. As before, from the genericity of the cycle 
$x+y+z$, we can suppose $Y$ free from non-trivial automorphisms and with very ample canonical bundle, $c f$. [C, Theorem F], [CFHR, Theorem 3.6].

From Proposition 8, we obtain a torsion-free, $\omega_{Y}$-semistable sheaf $F$ of rank one on $Y$ with $\chi(F)=1-k$ and $h^{0}(Y, F) \geq 2$. We show that $F$ yields either to a pencil of degree $k+1$ on $X$ which passes through $x, y$, and $z$ or to a pencil of degree at most $k$. Let $F_{E}$, and $F_{X}$ be the torsion-free parts of the restrictions of $F$ to $E$ and $X$, respectively. It is well-known that there is a natural injection $F \rightarrow F_{E} \oplus F_{X}$ whose cokernel is supported at the points among $x, y$ and $z$ where $F$ is invertible. We distinguish now four cases according to the number of nodes where $F$ is invertible.

(i) $F$ is invertible at all three $x, y$, and $z$.

In this case, $F_{\mid E}=F_{E}, F_{\mid X}=F_{X}$, and we have two exact sequences

$$
0 \rightarrow F_{E}(-3) \rightarrow F \rightarrow F_{X} \rightarrow 0
$$

and, respectively,

$$
0 \rightarrow F_{X}(-x-y-z) \rightarrow F \rightarrow F_{E} \rightarrow 0 .
$$

The subsheaves $F_{E}(-3)$ and $F_{X}(-x-y-z)$ are of multiranks $(1,0)$ and, respectively $(0,1)$, and, since $\operatorname{deg}\left(\omega_{Y \mid E}\right)=1$, and $\operatorname{deg}\left(\omega_{Y \mid X}\right)=2 g_{X}+1$, their $\omega_{Y}$-slopes are equal to

$$
\mu\left(F_{E}(-3)\right)=\chi\left(F_{E}(-3)\right)=\operatorname{deg}\left(F_{E}\right)-2,
$$

and, respectively,

$$
\mu\left(F_{X}(-x-y-z)\right)=\frac{\chi\left(F_{X}(-x-y-z)\right)}{2 g_{X}+1}=\frac{\operatorname{deg}\left(F_{X}\right)-2-g_{X}}{2 g_{X}+1},
$$

see, for example [P, Definition 1.1]. The $\omega_{Y}$-slope of $F$ equals

$$
\mu(F)=\frac{1-k}{2 g_{X}+2} .
$$

From the $\omega_{Y}$-semistability of $F$, we obtain $\operatorname{deg}\left(F_{E}\right)-2 \leq \mu(F)<0$, which implies $\operatorname{deg}\left(F_{E}\right) \leq 1$, and also $\left(\operatorname{deg}\left(F_{X}\right)-1-2 k\right) /(4 k-1) \leq(1-k) /(4 k)$, which shows that $\operatorname{deg}\left(F_{X}\right) \leq k+2$.

The first exact sequence implies $\chi\left(F_{X}\right)=\chi(F)-\chi\left(F_{E}(-3)\right)=3-k-\operatorname{deg}\left(F_{E}\right)$, so $\operatorname{deg}\left(F_{X}\right)=k+1-\operatorname{deg}\left(F_{E}\right)$.

Let us suppose $\operatorname{deg}\left(F_{X}\right)=k+2$, which implies $F_{E} \cong \mathscr{O}_{E}(-1)$. Then any global section of $F$ vanishes along $E$, and thus it vanishes at all the three points $x, y$, and $z$. Since $F$ has at least two sections, the sublinear system $H^{0}(F) \subset$ $H^{0}\left(F_{X}\right)$ on $X$ has $x, y$, and $z$ as base-points, in particular $h^{0}\left(F_{X}(-x-y-z)\right) \geq 2$. Then $X$ would carry a $g_{k-1}^{1}$, fact which contradicts the hypothesis.

Supposing now $\operatorname{deg}\left(F_{X}\right) \leq k+1$, from the first exact sequence we obtain $h^{0}\left(X, F_{X}\right) \geq h^{0}(Y, F) \geq 2$, and since $X$ does not carry a $g_{k}^{1}$, it follows that $F_{E}=\mathscr{O}_{E}$, and $F_{X}$ is a base-point-free $g_{k+1}^{1}$ on $X$. Let $\sigma$ be a non-zero global section of $F_{X}$ which vanishes at $x$; such a $\sigma$ exists as $h^{0}\left(X, F_{X}\right)=2$. Then $\sigma$ is the restriction of global section $\sigma_{0}$ of $F$, as the restriction morphism on global 
sections is in this case an isomorphism. Since $F_{E}=\mathscr{O}_{E}$, the restriction of $\sigma_{0}$ to $E$ is a constant function. But $\sigma_{0}$ vanishes at $x$, so it vanishes on the whole $E$. In particular, $\sigma_{0}$ vanishes at $y$ and $z$, and thus $\sigma$ vanishes at $y$ and $z$ as well. This is a contradiction, as we supposed that there was no such a $\sigma$.

(ii) $F$ is invertible at $y$, and $z$, and is not invertible at $x$.

Let $f: Z \rightarrow Y$ be the normalization of the point $x$. Then $F$ is the direct image of a line bundle $L$ on $Z$. Observe that $\chi(Z, L)=\chi(Y, F)=1-k$, and $h^{0}(Z, L)=h^{0}(Y, F) \geq 2$. We consider the exact sequences

$$
0 \rightarrow F_{E}(-2) \rightarrow L \rightarrow F_{X} \rightarrow 0,
$$

and, respectively,

$$
0 \rightarrow F_{X}(-y-z) \rightarrow L \rightarrow F_{E} \rightarrow 0,
$$

push them forward on $Y$, and argue as before. Since $\chi\left(F_{E}(-2)\right)=\chi\left(f_{*}\left(F_{E}(-2)\right)\right)$ (and the same for the other sheaf), we deduce, via the semistability of $F$, that $\operatorname{deg}\left(F_{E}\right) \leq 0$, and $\operatorname{deg}\left(F_{X}\right) \leq k+1$. The relation between the degrees of $F_{E}$ and $F_{X}$ is now $\operatorname{deg}\left(F_{X}\right)=k-\operatorname{deg}\left(F_{E}\right)$. Since $F_{E}$ can not be trivial (this would imply that $F_{X}$ is a $\left.g_{k}^{1}\right)$, this shows that $F_{E}$ can only be equal to $\mathscr{O}_{E}(-1)$, and $F_{X}$ is a $g_{k+1}^{1}$. Then any global section of $L$ will be identically zero on $E$, so it will vanish at $y$ and $z$. By the assumption on the gonality we obtain $h^{0}(Z, L)=h^{0}\left(X, F_{X}\right)=2$, which implies that any non-zero global section of $F_{X}$ vanishes at $y$, and $z$, so $y$ and $z$ are base points of the linear system $\left|F_{X}\right|$. Then $F_{X}(-y-z)$ is a $g_{k-1}^{1}$ on $X$, which contradicts the hypothesis.

(iii) $F$ is invertible at $x$, and is not invertible at $y$ and $z$.

Is similar to the previous case. Let $f: Z \rightarrow Y$ be the normalization of the points $y$ and $z$. Then $F$ is the direct image of a line bundle $L$ on $Z$ with $\chi(L)=\chi(F)=1-k$, and $h^{0}(Z, L)=h^{0}(Y, F) \geq 2$. We consider the exact sequences

$$
0 \rightarrow F_{E}(-1) \rightarrow L \rightarrow F_{X} \rightarrow 0
$$

and, respectively,

$$
0 \rightarrow F_{X}(-x) \rightarrow L \rightarrow F_{E} \rightarrow 0
$$

and prove that $\operatorname{deg}\left(F_{E}\right) \leq-1$, and $\operatorname{deg}\left(F_{X}\right) \leq k$. Then $F_{X}$ is a $g_{k}^{1}$ on $X$, contradiction.

(iv) $F \cong F_{E} \oplus F_{X}$.

In this case, since $F$ is semistable, on the one hand, we have $\chi\left(F_{E}\right) \leq \chi(F) /(4 k)$, and $\chi\left(F_{X}\right) \leq \chi(F)(4 k-1) /(4 k)$, and on the other hand $\chi\left(F_{E}\right)+\chi\left(F_{X}\right)=\chi(F)$. This implies that the two inequalities above must be equalities, which is actually impossible, since $(1-k) /(4 k)$ is not an integer if $k \geq 2$.

Remark 12. Stable curves with one smooth rational irreducible component have already been used in connection with the Green conjecture, cf. [Ei, Proof of Theorem 4], but with a somewhat different purpose. 


\section{Acknowledgements}

The present work emerged from some enlightening discussions with Claire Voisin, to whom I address my warmest thanks. I thank Fabrizio Catanese for having made pertinent remarks on the manuscript, and indicating me the veryampleness criteria for the canonical bundle of Gorenstein curves. Thanks also to Lucia Caporaso, Gavril Farkas, Frank Schreyer, Monserrat Teixidor i Bigas, for useful discutions on the topic, to the IHES Bures-sur-Yvette, where this work was started, and the University of Bayreuth, especially Thomas Peternell, for hospitality, and the Alexander von Humboldt Foundation for financial support.

\section{References}

[A1] M. Aprodu, On the vanishing of the higher syzygies of curves, Math. Z. 241 (2002), 1-15, II. Math. Z. 243 (2003), 775-778.

[A2] , Green-Lazarsfeld gonality conjecture for a generic curve of odd genus, Int. Math. Res. Not. 63 (2004), 3409-3416.

[AV] M. Aprodu and C. Voisin, Green-Lazarsfeld's conjecture for generic curves of large gonality, C. R. Math. Acad. Sci. Paris 336 (2003), 335-339.

[AC] E. Arbarello and M. Cornalba, Footnotes to a paper of Beniamino Segre, Math. Ann. 256 (1981), 341-362.

[ACGH] E. Arbarello, M. Cornalba, P. A. Griffiths and J. Harris, Geometry of Algebraic Curves, Grundlehren. math. Wiss. 267 (1985) Springer Verlag.

[Ca] L. Caporaso, A compactification of the universal Picard variety over the moduli space of stable curves, J. Amer. Math. Soc. 7 (1994), 589-660.

[C] F. Catanese, Pluricanonical-Gorenstein-curves, Enumerative geometry and classical algebraic geometry (Nice, 1981), 51-95, Progr. Math., 24, Birkhaeuser Boston, Boston, MA, 1982.

[CFHR] F. Catanese, M. Franciosi, K. Hulek and M. Reid, Embeddings of curves and surfaces, Nagoya Math. J. 154 (1999), 185-220.

[CP] C. Ciliberto and G. Pareschi, Pencils of minimal degree on curves on a K3 surface. J. Reine Angew. Math. 460 (1995), 15-36.

[CM] M. Coppens and G. Martens, Secant spaces and Clifford's theorem, Compositio Math. 78 (1991), 193-212.

[DM] P. Deligne and D. Mumford, The irreducibility of the space of curves of given genus, Publ. Math. I.H.E.S. 36 (1969), 75-109.

[Ei] L. Ein, A remark on the syzygies of the generic canonical curves, J. Diff. Geom. 26 (1987), 361-365.

[Eh] S. Ehbauer, Syzygies of points in projective space and applications, Zero-dimensional schemes (Ravello, 1992), 145-170, de Gruyter, Berlin, 1994.

[ELMS] D. Eisenbud, H. Lange, Herbert, G. Martens and F.-O. Schreyer, The Clifford dimension of a projective curve, Compositio Math. 72 (1989), 173-204.

[FHL] W. Fulton, J. Harris, R. Lazarsfeld, Excess linear series on an algebraic curve, Proc. Amer. Math. Soc. 92 (1984), 320-322.

[G] M. Green, Koszul cohomology and the geometry of projective varieties, J. Diff. Geom. 19 (1984), 125-171, with an Appendix by M. Green and R. Lazarsfeld.

[GL1] M. Green and R. Lazarsfeld, On the projective normality of complete linear series on an algebraic curve, Invent. Math. 83 (1985), 73-90.

[GL2] Special divisors on curves on a K3 surface, Invent. Math. 89 (1987), $357-370$.

[HM] J. Harris and D. Mumford, On the Kodaira dimension of the moduli space of curves, Invent. Math. 67 (1982), 23-86. 
[HR] A. Hirschowitz and S. Ramanan, New evidence for Green's conjecture on syzygies of canonical curves, Ann. Sci. École Norm. Sup. 31 (1998), 145-152.

[Ke] C. Keem, On the variety of special linear systems on an algebraic curve, Math. Ann. 288 (1990), 309-322.

[Lo] F. Loose, On the graded Betti numbers of plane algebraic curves, Manuscripta Math. 64 (1989), 503-514.

[GMa] G. Martens, On dimension theorems of the varieties of special divisors on a curve, Math. Ann. 267 (1984), 279-288.

[HMa] H. Martens, On the variety of special divisors. I, J. Reine Angew. Math. 227 (1967), 111-120.

$[\mathrm{Mu}]$ D. Mumford, Prym varieties. I, Contributions to analysis (a collection of papers dedicated to Lipman Bers), 325-350. Academic Press, New York, 1974.

[OS] T. Oda and S. Seshadri, Compactifications of the generalized jacobian variety, Trans. A.M.S. 253 (1979), 1-90.

[P] R. Pandharipande, A compactification over $\bar{M}_{g}$ of the universal moduli space of slope-semistable vector bundles, Journal of the A.M.S. 9 (1996), 425-471.

[S1] F.-O. Schreyer, Green's conjecture for general p-gonal curves of large genus, Algebraic curves and projective geometry (Trento, 1988), 254-260, Lecture Notes in Math., 1389, Springer, Berlin, 1989.

[S2] $\quad$ A standard basis approach to syzygies of canonical curves, J. Reine Angew. Math. 421 (1991), 83-123.

[T] M. Teixidor i Bigas, Green's conjecture for the generic $r$-gonal curve of genus $g \geq$ $3 r-7$, Duke Math. J. 111(2002), 195-222.

[V1] C. Voisin, Courbes tétragonales et cohomologie de Koszul, J. Reine Angew. Math. 387 (1988), 111-121.

[V2] Green's generic syzygy conjecture for curves of even genus lying on a K3 surface, J. Eur. Math. Soc. 4 (2002), 363-404.

[V3] , Green's canonical syzygy conjecture for generic curves of odd genus, Compositio Math. (2005) in press.

Institute of Mathematics "Simion Stollow" of the Romanian Academy P.O. Box 1-764 RO-014700 Bucharest Romania

E-mail address: Marian.Aprodu@imar.ro

Universität Bayreuth, Mathematisches Institut, Lehrstuhl Mathematik 1, D-95447 Bayreuth, Germany

E-mail address: aprodu@btm8x5.mat.uni-bayreuth.de 